\title{
Towards Interoperable and Reusable Learning Environments
}

\author{
Luis Anido, Martin Llamas and Mancel J. Fernández \\ Área de Ingenieria Telemática, Depto. Tecnologías de las Comunicaciones, ETSI \\ Telecomunicación, University of Vigo, Campus Universitario s/n, E-36200 Vigo, Spain \\ lanido@ait.uvigo.es
}

Keywords: authoring tools, courseware, lab-ware, CBT, ODL

\begin{abstract}
Learning technology standardisation is one of the key activities in the field of computer-based education. Eventually, this standardisation process will support resource sharing and interoperability. This paper presents an up-todate survey on this process, and then discusses our contribution to it. We have defined a distributed software architecture built on the CORBA middleware platform. The provided functionality is made available through software interfaces defined according to existing recommendations and standards on elearning. The architecture discussed can be implemented using traditional CORBA objects or CORBA Component Model (CCM) components.
\end{abstract}

\section{INTRODUCTION}

Computer-based learning platforms are usually developed ad-hoc to meet the requirements of a particular institution. Heterogeneous systems appear with no interoperability mechanism among them. Courses developed for a particular system cannot be reused by a different one. The students' performance profiles, as stored by a specific system, cannot be read or managed by a different educational platform. An evident interoperability problem exists in learning technology. A learning technology standardisation process has been started to overcome this heterogeneity problem. 
Most computer-based learning systems share some common functionality usually implemented from the scratch by each of them. The existence of reusable software elements to implement that functionality in a generic way would drastically reduce the time needed to develop a new computer-based educational system. The open definition of the interfaces provided by each component contributes to the interoperability among different systems and the standardisation process of learning technologies. Our work is mainly focused on this field. The first part of this paper is outlines the current trends in learning technology standardisation. The second part presents a CORBA (OMG 2001) architecture to develop scalable learning platforms making use of agreed recommendations on learning resources

\section{INTEROPERABILITY}

The main contributors to the learning technology standardisation are the following: IEEE's Learning Technology Standardisation Committee (LTSC 2001); IMS Global Learning Consortium (IMS 2001); Aviation Industry CBT Committee (AICC 2001); US Department of Defense's Advanced Distributed Learning initiative (ADL 2001); Alliance of Remote Instructional Authoring and Distribution Networks for Europe project (ARIADNE 2001); Getting Educational Systems Talking Across Leading Edge Technologies (GESTALT 2001); PROmoting Multimedia access to Education and Training in EUropean Society (PROMETEUS 2001); European Committee for Standardization Information Society Standardization System, Learning Technologies Workshop (CEN/ISSS/LT) (CEN 2001); and, Gateway to Educational Materials project (GEM 2001).

The IEEE's LTSC is the institution that is actually gathering recommendations and proposals from other learning standardisation institutions and projects. Specifications that have been approved by the IEEE go through a more rigorous process to become ANSI or ISO standards. In fact, a new ISO/IEC JTC1 Standards Committee for Learning Technologies, SC36 (JTC1 2001), was approved in November 1999. Below we present the main outcomes of these standardisation efforts obtained so far.

\subsection{Metadata for learning resources}

The learning metadata definition area has been one of the main activities for the learning standardisation community during the last few years. Metadata is just data about data, in this case, data about educational data and resources. The purposes of these definitions are, among others: to allow humans to search, evaluate, acquire and use learning objects, to enable 
sharing and exchanging of learning objects across any technology-supported learning system, let computer agents to automatically and dynamically compose personalised lessons for an individual learner, to enable educational institutions to express educational content and performance standards in a standardised format that is independent of the content itself. In short, it aims at the standardisation of learning resource description.

Important outcomes have been already delivered. One of the main contributors to this effort is the IEEE LTSC's (LTSC 2001) Learning Objects Metadata (LOM) working group. The LOM specification describes learning content cataloguing information. Relevant properties of learning objects include type of object, author, owner, terms of distribution, format, teaching or interaction style, grade, level, mastery level and prerequisites. The structured approach to metadata definition implies that the actual data elements of a learning resource are grouped into meaningful categories. The base LOM scheme consists of nine such categories: General, Lifecycle, Meta-metadata, Technical, Educational, Rights, Relation, Annotation and Classification.

LOM metadata is becoming a de-facto standard among the e-learning community. However, this specification is the result of the effort of many contributors, among them, the European ARIADNE project (ARIADNE 2001) and the IMS project (IMS 2001) stand out. ARIADNE uses LOM version 3.8 , to which it contributed significantly, to index and exploit its network of interconnected knowledge pools (KPS). The IMS Learning Resources Metadata Specifications (August 1999) is directly based on the IEEE's LOM with some changes based on implementation testing and detailed document reviews by the IMS Technical Board, which will probably be incorporated into the IEEE specifications. The IMS metadata specification identifies a minimum set of IEEE metadata elements called the IMS core (19 out of 86 LOM elements). The remaining IEEE metadata elements form the IMS Standard Extension Library, SEL, (67 out of 86 LOM elements). The IMS has also completed a survey to identify taxonomies and vocabularies, which can be used as values for the defined metadata elements.

The DoD's ADL (ADL 2001) Sharable Courseware Object Reference Model, applies the IEEE/IMS definitions to the three components of the SCORM model: raw media, content and courses. Other system that has extended the LOM definition is (GESTALT 2001), which delivered its own metadata specification: GEMSTONES. The main extensions of LOM include external rights management and the improvement of the quality of service description. GEMSTONES are used by the GESTALT brokerage service to locate learning resources. The Gateway to Educational Materials 
(GEM 2001) system also provides a brokerage service based on extensions of Dublin Core (DC 2001).

\subsection{Learner records and profiles}

Description of learner's profiles and records has also been studied in order to deliver recommendations on standards that allow the exchange of student data. The IEEE LTSC's (LTSC 2001) Public and Private Information (PAPI) specification describes portable and implementation-independent learner records. Learner records are organised into six major categories: personal, preference, performance, portfolio information, relations and security. They describe information about learners, about their technical, learning and physical preferences, about learners history and about their current works. Based on PAPI, the IMS (IMS 2001) Enterprise Data Model Specification (January 2000) is aimed at administrative services that need to share data about learners, courses, performance, etc., across platforms, Operating Systems, user interfaces and so on. This data model is supported through the use of three data objects: person, group and group membership.

\subsection{Course structure formats}

The US Department of Defense ADL initiative (ADL 2001), as part of its SCORM model, has identified an XML-based representation of a course structure format (CSF) that can be used to define all course elements, structure and external references necessary to move a course from one system to another. It is not course packaging, as the course structure format is just one (albeit very important) of the elements needed to move a course from a given system to another one. CSF describes a course using three groups of information. The first group, called globalProperties, is the data about the overall course. The second, called block, defines the structure of the course, and the third group, objectives, defines a separate structure for learning objectives with references to course elements within the assignment structure. Course sequencing is defined using prerequisites and completion requirements for blocks, assignable units and objectives. ADL's CSF is derived from the AICC (AICC 2001) file-based content model for course structures, properties, and objectives.

\section{$2.4 \quad$ Course packaging}

The IMS project (IMS 2001) is leading the standardisation process in this particular field. The IMS Content \& Packaging Specification (March 2000) 
makes it easier to create reusable learning resources. The key element is the package: an abstract description of a unit of reusable content. A package must provide all files and data needed to transfer the learning resources it embodies from one system to another. It is also possible to aggregate a package into a higher level one. The two components of a package are the manifest file and the physical resources. The manifest contains a metadata description of the package as a whole, one or more ways of organisation of the content, and can include or reference sub-manifests which describe the packages that have been included or referenced. The physical resources are a collection of resources physically included within a package.

\subsection{Questions and tests interoperability}

In February 2000, the IMS project has delivered the first specification on questions and test interoperability. It addresses the need to share test items and other assessment tools across different systems, and describes the data structures needed to provide interoperability between questions and test systems, particularly those that are Internet-based. The specification also defines a taxonomy that describes a set of response types and different forms for each of them.

\subsection{Learning run time environments}

Regarding the specification of concrete run time environments, the work by the AICC (AICC 2001) and the DoD's ADL (ADL 2001) stand out. The AICC guidelines for interoperability of Computer-Managed Instruction (CMI) systems and the ADL's SCORM based on the AICC specifications, deal with a common problem: in the past, authoring systems made the customer a captive of his own CMI system. In order to avoid this dependence between CMIs and CBTs (contents), a standard approach is defined to allow a single CMI system to initiate lessons from different CBT vendors. To accomplish this function, $\mathrm{CMI}$ and $\mathrm{CBT}$ must communicate by means of standard types of data: data from CMI to CBT to start the lesson, data from a CBT system to CMI needed to record student performance and assign the next learning unit, and data needed for evaluation of a lesson such as item response data, simulation performance data, etc.

Additionally, the GESTALT project (GESTALT 2001) identifies a run time architecture made up of components from previous ACTS projects: GAIA, Renaissance and Prospect. Business objects comprising the interfaces among the various software components within the GESTALT architecture are identified. Middleware solutions were used for this purpose: DCOM (DCOM 2001) for interfaces among systems to be run in the same institution 
and CORBA (CEN 2001) for those interfaces among systems from different institutions.

\section{A CORBA-BASED FRAMEWORK}

So far, the most outstanding results in the learning technologies standardisation area are from learning metadata, definitions of learner records and models, and course structure formats. Standardisation of the software that supports Computer-Managed Instruction or Learning Management Systems is more difficult to cope with. The next step should be to provide software that could be easily reused. Open interfaces would also allow interoperability at run time, even among systems from different institutions, to share not only learning resources, but also learning services. The AICC/ADL run time environments provide a clear and precise definition for the interfaces needed to manage educational resources. However, their interfaces do not describe a complete learning environment. GESTALT copes with the whole learning environment. They compose such kind of environment from the outcomes of three previous projects. Therefore, they only define interfaces between those elements belonging to different former projects. A greater level of detail and interfaces among objects from the same project are required. We need an interface definition as precise and detailed as the provided by AICC/ADL run time environments. But we also need to cover the whole learning spectrum, from the interaction between the learning contents and their management systems to a suitable location and brokerage tool for learning resources. We intend to define those interfaces using the CORBA (OMG 2001) technology.

We have defined a prospective CORBA domain interface to develop distributed learning environments using reusable elements instead of starting from scratch. These components have open interfaces that allow other objects, even from different systems, to access their services. In addition, the learning resources managed and delivered could be easily reused and localised as international format and metadata standards are used. Thus, interoperability both at business-logic and data level is achieved.

\subsection{CORBA Middleware}

CORBA (OMG 2001) is an object-based distributed architecture that allows distributed and heterogeneous applications to interoperate on a network. CORBA is a standard defined by more than 800 institutions that form the Object Management Group (OMG). Nowadays, the hot topic for the OMG is the definition of high level services, clearly oriented to a 
particular business domain. Examples of already existing domain interfaces are in the areas of Telecommunications, Manufacturing, Finance and Healthcare. They identify the objects needed, and their interfaces, in those domains to cover the needs of a wide range of requirements. Different vendors may change the implementation but the functionality is the same thanks to the agreed interface. Thus, CORBA domain interfaces contribute to the interoperability among systems. In addition, the OMG is an open consortium with no ties with particular software platforms or programming languages. Therefore, we chose CORBA as our middleware framework among other available options (e.g. DCOM (DCOM 2001)).

\subsection{Distributed architecture for learning environments}

The CORBA-based architecture for interoperable learning environments that we propose is outlined in Figure 1. Object interfaces are used as the basis for a computer-based education domain interface. Clients access learning services, through standard CORBA communication mechanisms, using the interfaces provided by Learning Server Objects. Two different interfaces have been defined: an AICC/ADL run time compliant interface and our Standard Run-time Interface (SRI). The latter defines standard mechanisms to interact with a learning environment (e.g. login, course index, next unit, previous unit, next assignment, etc.) and advanced features like predictive navigation (the object evaluates which learning resources are more likely to be requested next and sends them to the client side if their prerequisites were already fulfilled).

Navigation Manager objects could impose particular navigation sequences based on designer criteria and/or students' performance. So far, we defined interfaces for the AICC/ADL Course Structure Format (CSF) and a basic hierarchical structure with no prerequisites. Learning Server Objects use these interfaces to evaluate the next learning unit to be sent to a student, to check the prerequisites to access a unit or to resume student status in a particular course from stored data about previous learning sessions.

Students' performance tracking is done by Student Trackers who receive information from Learning Server Objects through Student Tracking channels based on the CORBA event service. Trackers are responsible for storing tracking information and providing later access to it. AICCcompliant and PAPI-compliant interfaces have been already defined. Performance Information at a higher level becomes grading information. Trackers report Administration objects whenever they detect students have fulfilled all needed requisites to pass a course. A CORBA event channel, Student Grading, is used to send this information from Trackers to Administration Objects, which update students' records. So far, 
administrative data are stored, accessed and managed using a PAPIcompliant interface. Learning Server Objects may use PAPI preference information to fit their behaviour to each student.

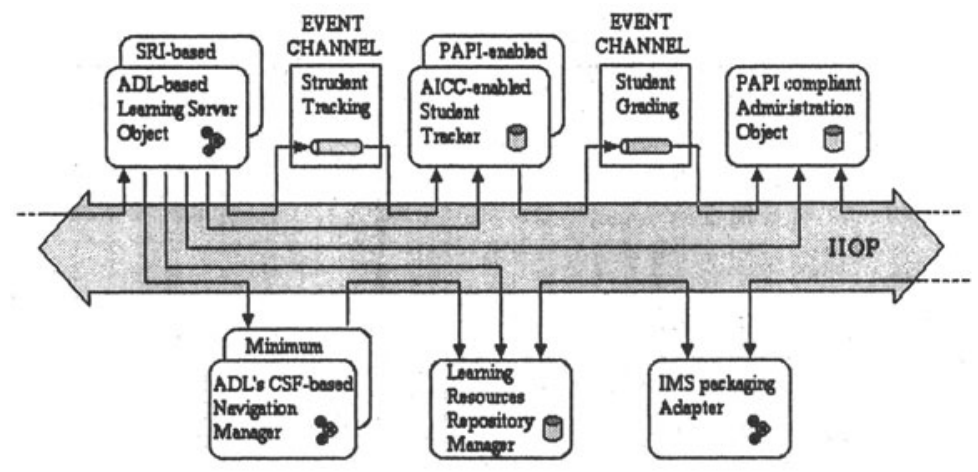

Figure 1. A CORBA-based architecture for Interoperable Learning Environments

The Learning Resources Repository Manager is responsible for making transparent for the rest of the system the particular storage mechanism. Both learning contents and metadata is stored by this object and accessed through it. The IMS Packaging Adapter defines through its interface operations to create and aggregate learning packages using the IMS packaging specification. It also provides operations to extract packaged data. These services are accessed following CORBA standard mechanisms. Defined objects do not impose restrictions regarding their physical location. They may run all of them in the same computer or distributed over a local network or over the Internet. Communication mechanisms are based on IIOP protocol that relies on TCP/IP standard protocol.

\subsection{Definition using the CORBA components model}

Defined objects could be implemented following the forthcoming CORBA Components Model (CCM) (OMG 2001), which is part of the CORBA 3.0 specification. The CCM extends the traditional CORBA object model by defining features and services that allow application programmers to implement, manage, configure and deploy software components that integrate commonly used CORBA services. The CCM standard not only enables greater software reuse but it also provides greater flexibility for dynamic configuration of CORBA applications. A component is a basic CORBA meta-type that can be referenced by multiple object references. Components can also interact with external entities through a rich set of port mechanisms. The architecture presented in Figure 1 can be implemented 
through a components model as shown in Figure 2 (the figure uses the CCM graphic symbols (OMG 2001)).

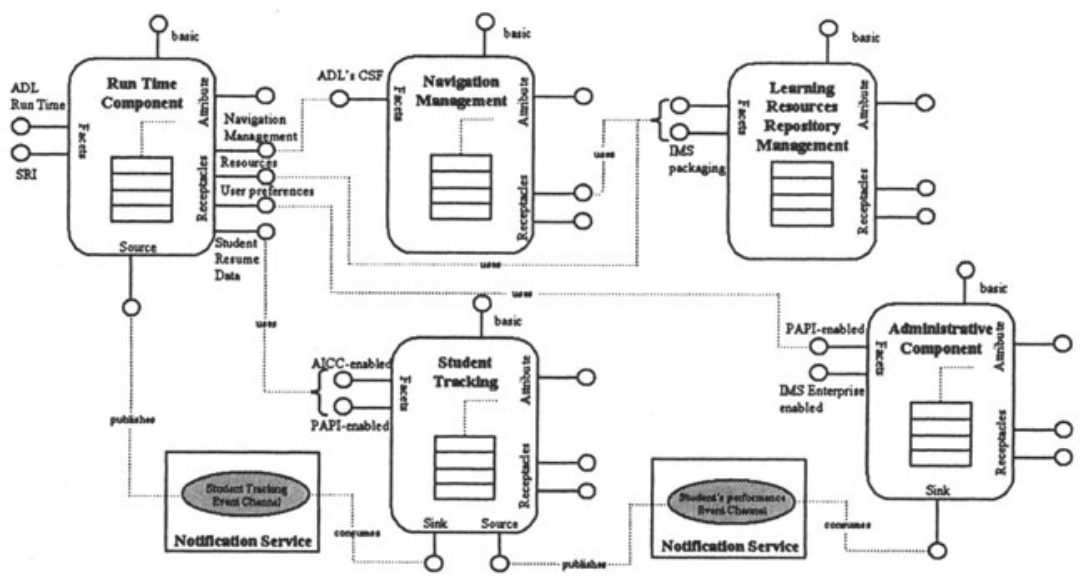

Figure 2. CORBA Components Model for the interoperable and reusable learning architecture

In this case, the same component offers different facets to provide the same functionality that was implemented by different objects in the traditional CORBA model. Communication among components is directly carried out through receptacles and event sources/sinks. These features, together with the deployment capabilities offered by the CCM, seems to make it a suitable framework to develop this kind of distributed systems. Unfortunately, at the time of this writing no CORBA implementation supports the CCM.

\section{CONCLUSIONS}

The CORBA architecture for computer-based learning allows the development and the deployment of distributed learning systems using reusable software elements. Scalability is fully provided because objects can be installed individually and later connected to other components to provide a more refined functionality (e.g. simple or performance-based navigation, single or federated searches, etc.). Developers do not need to start from scratch because defined objects are reusable. Therefore, the development and deployment process is improved and can be carry out quicker. The use of the CCM will notably improve the reusability of the defined objects (components). We present object interfaces as open definitions for object behaviours that, together with the use of internationally agreed formats for learning data and metadata (an up-to-date survey on this area appears in the 
first part of this paper) make our model a full interoperable framework for distributed computer-based learning systems. This leads to the possibility of using these interfaces as a new CORBA domain interface. Currently, we are also defining the interfaces for a learning brokerage system. This CORBAbased search tool allows one to locate, evaluate and retrieve educational resources.

\section{REFERENCES}

ADL (2001) DoD's Advanced Distributed Leaming. [http://www.adlnet.org] 23.4.2001. AICC (2001) Aviation Industry CBT Committee. [http://www.aicc.org] 23.4.2001. ARIADNE (2001) ARIADNE. [http://ariadne.unil.ch] 23.4.2001.

CEN (2001) CEN/ISSS/LT. [http://www.cenorm.be/isss/Workshop/lt] 23.4.2001.

DC (2001) Dublin Core metadata. [http://purl.org/dc/] 23.4.2001.

DCOM (2001) Microsoft's Distributed COM (DCOM).

[http://www.microsoft.com/com/tech/dcom.asp] 23.4.2001.

GEM (2001) GEM project. [http://www.geminfo.org] 23.4.2001.

GESTALT (2001) GESTALT. [http://www.fdgroup.co.uk/gestalt] 23.4.2001.

IMS (2001) IMS Global Learning Consortium. [http://www.imsproject.org] 23.4.2001.

JTC1 (2001) ISO-IEC JTC1 SC36. [http://www.jtc1sc36.org] 23.4.2001.

LTSC (2001) IEEE's LTSC. [http://tsc.ieee.org] 23.4.2001.

OMG (2001) OMG's CORBA. [http://www.omg.org ] 23.4.2001.

PROMETEUS (2001) PROMETEUS. [http://prometeus.org] 23.4.2001.

\section{BIOGRAPHY}

Luis Anido works at the Telecommunication Engineering Faculty of the University of Vigo where he is working towards his $\mathrm{PhD}$. His main areas of interest are the standardisation of learning technologies and distributed computing. 